\title{
Enteral glutamine supplementation in critically ill patients: a systematic review and meta-analysis
}

\author{
Arthur R. H. van Zanten', Rupinder Dhaliwal ${ }^{2}$, Dominique Garrel ${ }^{3}$ and Daren K. Heyland ${ }^{2 *}$
}

\begin{abstract}
Introduction: Glutamine (GLN) has been suggested to have a beneficial influence on outcomes of critically ill patients. However, recent large-scale trials have suggested harm associated with GLN supplementation. Recently, systematic reviews on the use of parenteral GLN have been published; however, less information is available on the role of enteral GLN. Therefore, the aim of this systematic review was to study the effects of enteral GLN supplementation in patients with critical illness.
\end{abstract}

Methods: We identified randomized controlled trials conducted from 1980 to 2014 with enterally administered GLN in adult critically ill patients. Studies of parenteral GLN only or combined enteral-parenteral GLN were excluded. The methodological quality of studies was scored, and trial data were statistically combined. We examined a priori the treatment effects in subgroups of trials of burn and trauma patients.

Results: A total of 11 studies involving 1079 adult critically ill patients and enteral GLN supplementation were identified. Enteral GLN supplementation was not associated with a reduction of hospital mortality (risk ratio [RR] $0.94,95 \%$ confidence interval [Cl] 0.65-1.36; $p=0.74$ ), infectious complications (RR 0.93, $95 \% \mathrm{Cl} 0.79-1.10 ; p=0.39$ ) or stay in the intensive care unit (weighted mean difference [WMD] -1.36 days, $95 \% \mathrm{Cl}-5.51$ to $2.78 ; p=0.52$ ). However, there was a significant reduction in hospital stay (WMD 4.73 days, $95 \% \mathrm{Cl}-8.53$ to $-0.90 ; p=0.02$ ). In the subset of studies of patients with burns, enteral GLN supplementation was associated with significant reductions in hospital mortality (RR 0.19, $95 \% 0.06-0.67 ; p=0.010$ ) and hospital stay (WMD $-9.16,95 \% \mathrm{Cl}-15.06$ to -3.26 ; $p=0.002$ ). There was no effect in trauma patients.

Conclusions: Enteral GLN supplementation does not confer significant clinical benefit in critically ill patients, with the exception of reduced hospital stay. There may be a significant benefit in patients with burns, but data are sparse and larger randomized trials are warranted to confirm this effect.

\section{Introduction}

Immune-modulating nutrients are considered to modulate inflammatory and oxidative stress responses and to optimize the impaired (cellular) immune function [1]. Glutamine (GLN) is the most abundant free (non-essential) amino acid of the 20 amino acids in humans. No deficiencies are likely to be present in healthy persons, as GLN can be synthesized de novo. However, in catabolic and stress states that are commonly present in critically ill, trauma and burn patients, low plasma levels of

\footnotetext{
* Correspondence: dkh2@queensu.ca

${ }^{2}$ Clinical Evaluation Research Unit, Kingston General Hospital, Angada 4, 76 Stuart Street, Kingston, ON K7L 2V7, Canada

Full list of author information is available at the end of the article
}

GLN upon intensive care unit (ICU) admission have been encountered, leading to the assumption that these levels are too low for the actual medical condition and that GLN should be considered conditionally deficient [2]. The metabolic effects of GLN suggest a role in the inflammatory and oxidative stress responses [3]. On the basis of the association of low plasma GLN levels $(<420 \mu \mathrm{mol} / \mathrm{L})$ upon ICU admission and increased hospital mortality, one study group suggested that GLN supplementation in critically ill patients could be essential [4].

Since then, many studies on parenteral and/or enteral GLN supplementation in critically ill patients have been performed, with the earliest of these published in 1997 [5]. The authors of several older systematic reviews and 
meta-analyses reported that GLN supplementation, combined with enteral nutrition (EN) and parenteral nutrition $(\mathrm{PN})$, is associated with reduced infectious morbidity and improved recovery from critical illness compared with standard nutrition [6-8].

Results of earlier meta-analyses were based mainly on small, single-center studies. This led to the development of international guidelines for the use of enteral GLN in critically ill patients [9-11].

However, in the latest meta-analysis published by the Cochrane Database of Systematic Reviews, signals for mortality reduction were lost and only moderate- and low-level evidence on reduction of morbidity was found, with high risk of overall bias, suspected publication bias and moderate to substantial heterogeneity within the included studies [12].

Most recently, two large, multicenter studies-the Reducing Deaths Due to Oxidative Stress (REDOXS) and MetaPlus trials-have shown no effects of GLN on infectious morbidity; however, more importantly, increased long-term mortality in critically ill patients in the GLN supplementation arms was demonstrated [13, 14]. Together, the results of these two studies challenge current guidelines and recommendations for enteral and/or parenteral GLN in critically ill patients, as safety concerns have been communicated [15].

An up-to-date review on parenteral use of GLN has become available recently [16]; however, no systematic analysis focused on enteral GLN supplementation in critical illness has been performed over the last 6 years [17]. Moreover, a similar dose of GLN or glutaminealanine dipeptide administered through the enteral versus the parenteral route has smaller effects on plasma GLN levels, possibly owing to splanchnic extraction [18]. Furthermore, investigators in many trials on PN supplemented with GLN (typically without GLN in the control product) have studied patients not receiving EN. In contrast, in the control groups in the EN GLN studies, the standard commercially available EN typically contains limited amounts of GLN (5-6 g/L). Therefore, available data on parenteral GLN supplementation cannot be extrapolated to EN supplementation and thus may not be used as a basis for recommendations for enteral GLN administration.

In the present systematic literature review and metaanalysis, we address the question whether enteral administration of GLN as part of nutrition support has a positive effect on clinical outcomes in general, trauma and burn injury patients who were critically ill.

\section{Methods}

\section{Study identification}

The following databases were searched for articles published between 1980 and September 2014: Embase, MEDLINE, CINAHL, the Cochrane Central Register of Controlled Trials and the Cochrane Database of
Systematic Reviews. In the literature search, we used broad search terms containing "randomized," "blind," "clinical trial," "nutrition," "nutritional support" or "dietary supplementation" or "enteral nutrition" or "parenteral nutrition" or "parenteral nutrition solutions" and "critical care" or "critical illness" or "intensive care unit". The results were then reviewed to identify articles describing enteral GLN supplementation. A unique feature of this meta-analysis is that no language restrictions were placed on the searches. The authors personal files and reference lists of relevant review articles were also reviewed. As this is a systematic review, no ethics board approval or patient consent was required.

\section{Study selection criteria}

We included original studies only if they met the following inclusion criteria:

1. Study design: randomized clinical trials.

2. Study population: critically ill adult patients ( $>18$ years of age), defined as patients admitted to an ICU. When this information was unclear, we considered a mortality rate higher than $5 \%$ (hospital mortality or, if this was not reported, ICU mortality or 28-day mortality) in the control group to be consistent with critical illness.

3. Intervention: enteral GLN versus control (isonotrogenous control).

4. Study outcomes: must have included one of the following: mortality, ICU and hospital lengths of stay (LOSs) and infectious complications.

Studies in which the authors reported only other clinical endpoints, such as duration of mechanical ventilation, and studies of parenteral GLN only or combined enteral and parenteral GLN were excluded.

\section{Data abstraction}

Decisions about the inclusion of the articles were made in duplicate. Two reviewers, using a data abstraction form with a scoring system, reviewed all original studies independently. An assessment of the criteria for inclusion, details on the patient population, intervention and control and/or placebo, and clinical outcomes was done as described in earlier publications [19]. Using a scoring system we previously developed, we assessed the methodological quality of individual trials according to (1) whether randomization was concealed, (2) blinding, (3) whether the analysis was based on the intention-to-treat principle, (4) patient selection, (5) comparability of groups at baseline, (6) extent of follow-up, (7) description of treatment protocol and cointerventions and (8) definition of clinical outcomes [19]. Each individual study was scored from 1 to 14 (Table 1). Disagreement regarding the 
Table 1 Study scoring data abstraction form used to score all original studies independently

\begin{tabular}{|c|c|c|c|}
\hline & \multicolumn{3}{|l|}{ Score } \\
\hline & 0 & 1 & 2 \\
\hline Randomization & - & Not concealed or not sure & Concealed randomization \\
\hline Analysis & Other & - & Intention to treat \\
\hline Blinding & Not blinded & Single-blind & Double-blind \\
\hline Patient selection & Selected patients or unable to tell & Consecutive eligible patients & - \\
\hline Comparability of groups at baseline & No or not sure & Yes & - \\
\hline Extent of follow-up & $<100 \%$ & $100 \%$ & - \\
\hline Treatment protocol & Poorly described & Reproducibly described & - \\
\hline Cointerventions & Not described & Described but not equal or not sure & Well described and all equal \\
\hline Outcomes & Not described & Partially described & Objectively defined \\
\hline
\end{tabular}

individual scores of each of the categories was resolved by consensus between the two reviewers. We attempted to contact the authors of included studies and requested additional information not contained in their published articles.

\section{Data synthesis}

The primary outcome of the systematic review was hospital mortality. From all studies, we extracted data regarding hospital mortality if reported (specified or assumed to be hospital mortality if not specified). If hospital mortality was not reported, we used ICU mortality or 28-day mortality. Secondary outcomes included infection and ICU and hospital LOSs. We used definitions of infections as defined by the authors of the original articles. We combined data from all trials to estimate the pooled risk ratio (RR) with $95 \%$ confidence interval (CI) for mortality and infectious complications and overall weighted mean difference (WMD) with $95 \%$ CI for LOS data. Pooled RRs were calculated using the MantelHaenszel test, and WMDs were estimated using the inverse variance approach. The random-effects model of DerSimonian and Laird was used to estimate variances for the Mantel-Haenszel and inverse variance estimations [20]. All analyses except the test for asymmetry were conducted using Review Manager (RevMan) 5.1 software [21].

When possible, studies were aggregated on an intentionto-treat basis (Table 2). The presence of heterogeneity was tested using a weighted Mantel-Haenszel $\chi^{2}$ test and quantified by the $I^{2}$ statistic as implemented in RevMan 5.1 [21, 22]. Upon review of the dataset, we found that one randomized controlled trial contained other supplemental nutrients, not just GLN. To evaluate the effect of that trial on the overall results, we performed a sensitivity analysis wherein we excluded the trial to see how it affected the overall results [14]. The possibility of publication bias was assessed by generating funnel plots and testing asymmetry of outcomes using methods proposed by Rucker and colleagues [23]. We considered $p<0.05$ to be statistically significant and $p<0.20$ as the indicator of trend.

Table 2 Included randomized studies of enteral glutamine supplementation in critically ill patients

\begin{tabular}{|c|c|c|c|c|c|c|c|}
\hline Author & Year & ICU population & Setting & All patients & GLN+ patients & GLN- patients & Reference \\
\hline Houdijk et al. & 1998 & Critically ill trauma (100 \%) & Single center & 80 & 41 & 39 & {$[24]$} \\
\hline Jones et al. & 1999 & Mixed ICU (6 burns, 6 trauma) & Single center & 50 & 26 & 24 & {$[25]$} \\
\hline Brantley and Pierce & 2000 & Critically ill trauma (100%) & Single center & 72 & 31 & 41 & {$[26]$} \\
\hline Hall et al. & 2003 & Mixed ICU (mostly trauma, 7 burn) & Single center & 363 & 179 & 184 & {$[27]$} \\
\hline Garrel et al. & 2003 & Burns (TBSA: 20-80 \%) & Single center & 45 & 21 & 24 & {$[28]$} \\
\hline Zhou et al. & 2003 & Severe burns TBSA 50-80\% & Single center & 40 & 20 & 20 & {$[29]$} \\
\hline Peng et al. & 2004 & Severe burns TBSA > $30 \%$ & Single center & 48 & 25 & 23 & {$[30]$} \\
\hline Luo et al. & 2008 & Mixed ICU Medical-surgical & Single center & 30 & 15 & 15 & {$[31]$} \\
\hline McQuiggan et al. & 2008 & Shock trauma patients & Single center & 20 & 10 & 10 & {$[32]$} \\
\hline Pattanshetti et al. & 2009 & Burns (TBSA: 20-60 \%) & Single center & 30 & 15 & 15 & {$[33]$} \\
\hline van Zanten et al. & 2014 & Mixed ICU (109 trauma) & Multicenter & 301 & 152 & 149 & [14] \\
\hline
\end{tabular}




\section{Subgroup analyses}

We performed a predefined subgroup analysis to assess a number of possible influences on the effect of enteral GLN supplementation on clinical outcomes. We first explored whether there was a different treatment effect of enteral GLN in patients with burn injury and patients with trauma. The trial done by van Zanten and colleagues also contained an a priori subgroup analysis of patients with trauma, and we were able to obtain the data for the subset of trauma patients and include these data in the subgroup analysis [14]. We also assessed the effect of trial quality on outcome, as it is often hypothesized that, compared with trials of higher methodological quality, trials of lower methodological quality tend to yield more positive clinical signals for the therapy being tested. Using our trial scoring tool, we designated trials with a methodological score of 9 (out of a maximum score of 14) or more (median of scoring of all trials) as a high-quality trial for the purposes of this review.

\section{Results}

\section{Study identification and selection}

The literature search yielded 42 potentially eligible randomized controlled trials, of which 11 with a total of 1079 patients were included in our systematic review (see Table 2) [14, 24-33]. In total, 535 patients were treated with GLN supplementation and 544 patients with a control feed.

As shown in Table 3, a total of 33 studies [34-65] were excluded for the following main reasons: (1) patients not considered to be adult critically ill patients $(n=9)$; (2) no clinical outcomes meeting inclusion criteria $(\mathrm{n}=9)$; (3) being duplicate studies, reviews of published trials or subgroups of included studies $(\mathrm{n}=6)$; (4) crossover study design ( $\mathrm{n}=4$ ); and/or (5) multiple other interventions were studied, such as arginine, glycine, probiotics and fibers $(n=4)$.

Thus, we ultimately included 11 studies of enteral GLN supplementation performed in ICU patients with diagnoses ranging from trauma to burns and sepsis, as described in Table 3 [14, 24-33]. The results were based on data derived from the included studies, depicted in Table 4.

\section{Effect of enteral glutamine supplementation on hospital mortality}

When the data from 10 of the 11 total identified EN GLN studies that reported on mortality (Fig. 1) were aggregated, enteral GLN supplementation was not associated with a reduction in hospital mortality (RR 0.94, $95 \%$ CI $0.65-1.36 ; p=0.74$; test for heterogeneity $I^{2}=21 \%$ ). The combined hospital mortality was 79 (15.6\%) of 507 in the GLN group and 84 (16.3\%) of 515 in the control group. In the sensitivity analysis without the van Zanten et al. trial [14], there was still no effect on mortality (RR 0.80, $95 \%$ CI 0.46-1.38; $p=0.42$; heterogeneity $I^{2}=27 \%$ ). Also, in the subgroup of trauma patients, no effect on hospital mortality was found (RR 1.03, 95 \% CI 0.54-1.97; $p=0.92$; heterogeneity $I^{2}=0 \% ; \mathrm{n}=5$ studies). However, in the small subgroup of burn patients, a statistically significant reduction in mortality (2 [3.6\%] of 56 versus 14 [23.7\%] of 59) was demonstrated (RR $0.19,95 \% \mathrm{CI}$ $0.06-0.67 ; p=0.010$; heterogeneity $I^{2}=0 \%$; $=3$ studies).

\section{Effect of glutamine supplementation on infectious complications}

When the four studies in which the researchers reported infectious complications were aggregated, enteral GLN supplementation was not associated with a reduction in infectious complications (RR 0.93, $95 \%$ CI 0.79-1.10, $p=0.39$; heterogeneity $I^{2}=0 \%$ ) (Fig. 2). The overall incidence of infection was $140(36.3 \%)$ of 386 in the GLN group and 153 (39.2\%) of 390 in the control group. A sensitivity analysis without the van Zanten et al. study [14] showed a trend toward a reduction in infectious morbidity (RR $0.83,95 \%$ CI $0.64-1.08, p=0.16$; heterogeneity $I^{2}=0 \%$ ). Also, in the subgroup of trauma patients, a trend toward a reduction in infectious morbidity was found (RR $0.85,95 \%$ CI $0.68-1.06, p=0.15$; heterogeneity $I^{2}=0 \% ; \mathrm{n}=2$ studies). In the small subgroup of burn patients, few data on infections were available. Zhou et al. [29] reported infections in 2 (10\%) of 20 of burn patients treated with GLN versus $6(30 \%)$ of 20 in the control group. Garrel et al. [28] showed reductions in positive blood cultures in 7 (37 \%) of 19 in GLN-treated patients versus 10 (45 \%) of 22 in control subjects.

Effect of glutamine supplementation on ICU length of stay When we aggregated data from the three studies in which authors reported ICU LOS as mean \pm standard deviation (Fig. 3), we found that enteral GLN supplementation was not associated with a reduction in ICU LOS (WMD -1.36, $95 \%$ CI -5.51 to $2.78 ; p=0.52$; heterogeneity $\left.I^{2}=70 \%\right)$. When we excluded the van Zanten et al. study [14], we also observed no effect on ICU LOS (WMD -1.59 , $95 \%$ CI -8.15 to $4.96 ; p=0.63$; heterogeneity $\left.I^{2}=82 \%\right)$. In the small subgroup of trauma patients, we found a trend toward reduction in ICU LOS (WMD $-4.66,95 \%$ CI -9.68 to $0.36 ; p=0.07$; heterogeneity $I^{2}=82 \% ; \mathrm{n}=2$ studies). In the small number of trials with burn patients, no data on ICU LOS were available.

\section{Effect of glutamine supplementation on hospital length of stay}

When we aggregated the seven studies in which investigators reported data on hospital LOS (Fig. 4), we found 
Table 3 Excluded randomized studies of enteral glutamine supplementation in critically ill patients

\begin{tabular}{|c|c|c|c|}
\hline Author & Year & Reasons for exclusion & References \\
\hline Jebb et al. & 1995 & Transplant and/or elective surgery patients & {$[34]$} \\
\hline Long et al. & 1995 & No clinical outcomes & {$[35]$} \\
\hline Jensen et al. & 1996 & No clinical outcomes & {$[36]$} \\
\hline Fish et al. & 1997 & Cancer patients & {$[37]$} \\
\hline Scolapio et al. & 1997 & Crossover design & [38] \\
\hline Anderson et al. & 1998 & Surgical patients & [39] \\
\hline Anderson et al. & 1998 & Pediatric cancer patients & [40] \\
\hline Den Hond et al. & 1999 & Not ICU patients & [41] \\
\hline Schloerb and Skikne & 1999 & Cancer and/or surgery patients & [42] \\
\hline Scolapio & 1999 & Crossover design & [43] \\
\hline Zhou et al. & 1999 & Earlier study of 2003 RCT already included & [44] \\
\hline Jackson et al. & 2000 & Surgery patients, no clinical outcomes & {$[45]$} \\
\hline Szkudlarek et al. & 2000 & Crossover design & [46] \\
\hline Chen et al. & 2001 & No clinical outcomes & [47] \\
\hline Scolapio et al. & 2001 & Crossover design & [48] \\
\hline Velasco et al. & 2001 & No clinical outcomes, duplicate of Houdijk et al. study [24] & [49] \\
\hline Boelens et al. & 2002 & No clinical outcomes & [50] \\
\hline Novak et al. & 2002 & Studies on critically ill patients were included in this review & [51] \\
\hline Flaring et al. & 2003 & Elective surgery patients & [52] \\
\hline García-de-Lorenzo et al. & 2003 & Systematic review, Individual studies were included in this review & [53] \\
\hline Boelens et al. & 2004 & Duplicate of Houdijk et al. study [24] & [54] \\
\hline Falcao de Arruda et al. & 2004 & Includes probiotics & {$[55]$} \\
\hline Peng et al. & 2005 & Duplicate study of earlier publication already [30] included & {$[56]$} \\
\hline Peng et al. & 2006 & Duplicate of a previous study [30] & [57] \\
\hline Guo et al. & 2007 & No clinical outcomes & [58] \\
\hline Kuhls et al. & 2007 & Too many interventions & [59] \\
\hline Spindler-Vesel et al. & 2007 & Too many interventions: RCT of GLN vs. fiber vs. peptide vs. fiber + synbiotics & [60] \\
\hline Beale et al. & 2008 & Non-isonitrogenous intervention including arginine and glycine & [61] \\
\hline Han et al. & 2012 & Elective surgery patients & {$[62]$} \\
\hline Cavalcante et al. & 2012 & No clinical outcomes, crossover design & {$[63]$} \\
\hline Han et al. & 2014 & No clinical outcomes & [64] \\
\hline Koksal et al. & 2014 & Only duration of mechanical ventilation reported & [65] \\
\hline
\end{tabular}

GLN glutamine, ICU intensive care unit, $R C T$ randomized controlled trial

that GLN supplementation was associated with a significant reduction in hospital LOS (WMD -4.73, $95 \%$ CI -8.56 to $-0.90 ; p=0.02$; heterogeneity $I^{2}=52 \%$ ). The finding of a significant reduction in hospital LOS persisted after we excluded the van Zanten et al. study [14] (WMD 6.95 days, $95 \% \mathrm{CI}-12.37$ to -1.53 ; $p=0.01$; heterogeneity $I^{2}=76 \%$ ). In the subgroup of trauma patients, no reduction in hospital LOS was found (WMD -0.54 , $95 \% \mathrm{CI}-4.40$ to $3.31, p=0.78$; heterogeneity $I^{2}=0 \%$; $\mathrm{n}=4$ studies). In the small subgroup of burn patients, we found a significant reduction in hospital LOS (WMD -9.16, $95 \%$ CI -15.06 to -3.26 ; $p=0.002$; heterogeneity $I^{2}=52 \%$; n $=3$ studies).

\section{Effect of study quality on outcomes}

There was no effect of enteral GLN on reduction in hospital mortality in high-quality trials (RR 0.90, $95 \%$ CI 0.551.48; $p=0.69$ ) compared with low-quality trials (RR 0.84 , $95 \%$ CI $0.28-2.50 ; p=0.75$ ) when we tested for subgroup differences ( $p=0.90$; data not shown). There was an insufficient number of trials in which authors reported data on infectious outcomes and LOS in the low- and high-quality trial categories to allow for these comparisons to be made.

\section{Risk of publication bias across studies}

Funnel plots for all outcomes were created to assess for publication bias (data not shown). The test of asymmetry 
Table 4 Relevant outcome parameters of included randomized studies of enteral glutamine supplementation in critically ill patients

\begin{tabular}{|c|c|c|c|c|c|c|c|c|c|c|}
\hline \multirow[t]{3}{*}{ Study } & \multirow{3}{*}{$\begin{array}{l}\text { Methods } \\
\text { Score }\end{array}$} & \multirow{3}{*}{$\begin{array}{l}\text { Intervention } \\
\text { Dose (g/kg/day) } \\
\text { Type of feeding }\end{array}$} & \multicolumn{2}{|c|}{ Mortality, n (\%) ${ }^{a}$} & \multicolumn{2}{|c|}{ Infections, n (\%) ${ }^{b}$} & \multicolumn{2}{|c|}{ Hospital stay (days) } & \multicolumn{2}{|l|}{ ICU LOS (days) } \\
\hline & & & \multirow[t]{2}{*}{ Experimental } & \multirow[t]{2}{*}{ Control } & \multirow[t]{2}{*}{ Experimental } & \multirow[t]{2}{*}{ Control } & \multirow[t]{2}{*}{ Experimental } & \multirow[t]{2}{*}{ Control } & \multirow[t]{2}{*}{ Experimental } & \multirow[t]{2}{*}{ Control } \\
\hline & & & & & & & & & & \\
\hline \multirow{4}{*}{$\begin{array}{l}\text { Houdijk } \\
\text { et al. [24] }\end{array}$} & C. random: Yes & $>0.25$ & \multirow[t]{4}{*}{ 4/41 (9.8) } & \multirow[t]{4}{*}{$3 / 39(7.7)$} & \multirow[t]{4}{*}{ 20/35 (57.1) } & \multirow[t]{4}{*}{ 26/37 (70.2) } & \multirow[t]{4}{*}{$32.7 \pm 17.1$} & \multirow[t]{4}{*}{$33.0 \pm 23.8$} & \multirow[t]{4}{*}{ NA } & \multirow[t]{4}{*}{ NA } \\
\hline & ITT: No & \multirow{2}{*}{$\begin{array}{l}\text { Altira Q (glutamine-enriched } \\
\text { formula) vs. isonitrogenous } \\
\text { control (added amino acids) }\end{array}$} & & & & & & & & \\
\hline & Blinding: Yes & & & & & & & & & \\
\hline & 10 & $\begin{array}{l}\text { Same volume of feeding } \\
\text { received in both groups }\end{array}$ & & & & & & & & \\
\hline \multirow{6}{*}{$\begin{array}{l}\text { Jones } \\
\text { et al. [25] }\end{array}$} & C. random: Yes & 0.16 & Hospital & Hospital & NA & NA & NA & NA & $11(4-54)$ & $16.5(5-66)$ \\
\hline & ITT: No & \multirow{5}{*}{$\begin{array}{l}\text { Protina Torre MP (Fresenius } \\
\text { Kabi, Bad Homburg, } \\
\text { Germany) + glutamine } \\
\text { (10-15 g/day nitrogen) vs. } \\
\text { isonitrogenous control } \\
\text { (11-14 g/day nitrogen) }\end{array}$} & $10 / 26(38.5)$ & $9 / 24(37.5)$ & & & & & & \\
\hline & Blinding: Yes & & ICU & $\mathrm{ICU}$ & & & & & & \\
\hline & \multirow[t]{3}{*}{8} & & $9 / 26(35)$ & 9/24 (38) & & & & & & \\
\hline & & & 6 months & 6 months & & & & & & \\
\hline & & & $12 / 26(46)$ & 10/24 (42) & & & & & & \\
\hline Brantley and & C. random: Not sure & 0.50 & $0 / 31(0.0)$ & $0 / 41(0.0)$ & NA & NA & $19.5 \pm 8.8$ & $20.8 \pm 11.5$ & 11.4 & 11.1 \\
\hline & ITT: No & Glutamine-supplemented & & & & & & & & \\
\hline & Blinding: No & $\begin{array}{l}\text { enteral formula vs. Standard } \\
\text { formula (isonitrogenous) }\end{array}$ & & & & & & & & \\
\hline & 4 & protein given $1.5 \mathrm{~g} / \mathrm{kg} /$ day & & & & & & & & \\
\hline Hall et al. [27] & C. random: Yes & 0.27 & Hospital & Hospital & $38 / 179(21)$ & 43/184 (23) & $25(16-42)^{c}$ & $30(19-45)^{c}$ & $11(7-19)$ & $13(8-19)$ \\
\hline & ITT: Yes & Isocal (Nestlé Health Science, & 24/179 (13) & 23/184 (13) & & & & & $\begin{array}{l}\text { (exciuding } \\
\text { deaths) }\end{array}$ & $\begin{array}{l}\text { (exciuding } \\
\text { deaths) }\end{array}$ \\
\hline & Blinding: Yes & (66 g/day protein) vs. & ICU & ICU & & & & & & \\
\hline & 13 & isonitrogenous formula Isocal + & 16/179 (9) & 14/184 (8) & & & & & & \\
\hline & & & 30 days & 30 days & & & & & & \\
\hline & & & 26/179 (15) & 25/184 (14) & & & & & & \\
\hline & & & 6 months & 6 months & & & & & & \\
\hline & & & 27/179 (15) & 30/184 (16) & & & & & & \\
\hline Hall et al. [27] & C. random: Yes & 0.27 & 7/76 (9) & 6/78 (8) & Sepsis & Sepsis & NA & NA & NA & NA \\
\hline Trauma & ITT: Yes & Isocal + glutamine (66 g/day & & & 7/76 (9) & 11/78 (14) & & & & \\
\hline sul & Blinding: Yes & formula Isocal + glycine & & & & & & & & \\
\hline & 13 & (64 g/day protein) & & & & & & & & \\
\hline
\end{tabular}


Table 4 Relevant outcome parameters of included randomized studies of enteral glutamine supplementation in critically ill patients (Continued)

\begin{tabular}{|c|c|c|c|c|c|c|c|c|c|c|}
\hline \multirow[t]{4}{*}{$\begin{array}{l}\text { Garrel } \\
\text { et al. [28] }\end{array}$} & C. random: Yes & 0.28 & $2 / 21(10)$ & $12 / 24(50)$ & $\begin{array}{l}\text { Positive blood } \\
\text { cultures }\end{array}$ & $\begin{array}{l}\text { Positive blood } \\
\text { cultures }\end{array}$ & $33 \pm 17(16)^{d}$ & $29 \pm 17(19)^{d}$ & NA & NA \\
\hline & ITT: yes & \multirow{3}{*}{$\begin{array}{l}\text { Sandosource (Nestlé Health } \\
\text { Science) + glutamine } \\
(2.15 \mathrm{~g} / \mathrm{kg} / \text { day protein) vs. } \\
\text { Sandosource + amino acids } \\
\text { (isonitrogenous), } 1.97 \mathrm{~g} / \mathrm{kg} / \text { day } \\
\text { protein }\end{array}$} & & & 7/19 (37) & $10 / 22(45)$ & & & & \\
\hline & Blinding: Yes & & & & & & & & & \\
\hline & 11 & & & & & & & & & \\
\hline \multirow{4}{*}{$\begin{array}{l}\text { Zhou } \\
\text { et al. [29] }\end{array}$} & C. random: Yes & 0.35 & $0 / 20$ & $0 / 20$ & $2 / 20(10)$ & $6 / 20(30)$ & $67 \pm 4(20)$ & $73 \pm 6(20)$ & NA & NA \\
\hline & ITT: No & \multirow{3}{*}{$\begin{array}{l}\text { Ensure (NutriDrinks, Perivale, } \\
\text { UK) + glutamine vs. Ensure + } \\
\text { amino acids (isonitrogenous) }\end{array}$} & & & & & & & & \\
\hline & $\begin{array}{l}\text { Blinding: } \\
\text { Double-blind }\end{array}$ & & & & & & & & & \\
\hline & 8 & & & & & & & & & \\
\hline \multirow[t]{4}{*}{$\begin{array}{l}\text { Peng } \\
\text { et al. [30] }\end{array}$} & $\begin{array}{l}\text { C. random: } \\
\text { Not sure }\end{array}$ & 0.5 & NA & NA & NA & NA & $46.6 \pm 12.9(25)$ & $55.7 \pm 17.4$ & NA & NA \\
\hline & ITT: Yes & \multirow{3}{*}{$\begin{array}{l}\text { Oral glutamine granules vs. } \\
\text { placebo (isocaloric, } \\
\text { isonitrogenous) } 2.0 \mathrm{~g} / \mathrm{kg} / \mathrm{day} \\
\text { protein }\end{array}$} & & & & & & & & \\
\hline & Blinding: No & & & & & & & & & \\
\hline & 7 & & & & & & & & & \\
\hline \multirow[t]{4}{*}{ Luo et al. ${ }^{\mathrm{e}}$ [31] } & $\begin{array}{l}\text { C. random: } \\
\text { Not sure }\end{array}$ & 0.32 & ICU & $\mathrm{ICU}$ & NA & NA & NA & NA & $8.1 \pm 0.4(12)$ & $6.9 \pm 0.9(9)$ \\
\hline & ITT: No & \multirow{2}{*}{$\begin{array}{l}\text { Glutamine + IV saline + vs. } \\
\text { Nutren (Nestlé Health } \\
\text { Science) + } 15 \% \text { Clinisol } \\
\text { (Baxter Healthcare, Deerfield, } \\
\text { IL, USA) (placebo) (isocaloric, } \\
\text { isonitrogenous) }\end{array}$} & $1 / 12$ & $0 / 9$ & & & & & & \\
\hline & $\begin{array}{l}\text { Blinding: } \\
\text { Double-blind }\end{array}$ & & 28 days & 28 days & & & & & & \\
\hline & 9 & $1.7 \mathrm{~g} / \mathrm{kg} / \mathrm{d}$ protein & $1 / 12$ & $0 / 9$ & & & & & & \\
\hline \multirow[t]{4}{*}{$\begin{array}{l}\text { McQuiggan } \\
\text { et al. [32] }\end{array}$} & $\begin{array}{l}\text { C. random: } \\
\text { Not sure }\end{array}$ & \multirow{4}{*}{$\begin{array}{l}0.5 \text { (actual 0.4) IMPACT } \\
\text { (Nestlé Health Science) + } \\
\text { Glutasolve (Nestlé Health } \\
\text { Science) via NJ tube } \\
(1.3 \mathrm{~g} / \mathrm{kg} / \text { day protein), bolus } \\
\text { with } \mathrm{H}_{2} \mathrm{O} \text { vs. Impact + protein } \\
\text { supplements (isonitrogenous, } \\
\text { isocaloric) } 0.85 \mathrm{~g} / \mathrm{kg} / \text { day protein }\end{array}$} & \multirow[t]{4}{*}{$0 / 10$} & \multirow[t]{4}{*}{ 2/10 (20) } & \multirow[t]{4}{*}{ NA } & \multirow[t]{4}{*}{ NA } & \multirow[t]{4}{*}{$32 \pm 13.6(10)$} & \multirow[t]{4}{*}{$39.3 \pm 33.6(10)$} & \multirow[t]{4}{*}{$4.8 \pm 6.7(10)$} & \multirow[t]{4}{*}{$10.4 \pm 6.2(10)$} \\
\hline & ITT: Yes & & & & & & & & & \\
\hline & Blinding: No & & & & & & & & & \\
\hline & 10 & & & & & & & & & \\
\hline \multirow[t]{4}{*}{$\begin{array}{l}\text { Pattanshetti } \\
\text { et al. [33] }\end{array}$} & $\begin{array}{l}\text { C. random: } \\
\text { Not sure }\end{array}$ & \multirow{4}{*}{$\begin{array}{l}\text { Enteral isonitrogenous } \\
\text { mixture + EN glutamine }+ \\
\text { "regular" nutrition vs. enteral } \\
\text { isonitrogenous mixture + } \\
\text { "regular" nutrition }\end{array}$} & \multirow[t]{4}{*}{$0 / 15$} & \multirow[t]{4}{*}{$2 / 15$} & \multirow{3}{*}{$\begin{array}{l}\text { Number of } \\
\text { times positive } \\
\text { blood cultures }\end{array}$} & \multirow{3}{*}{$\begin{array}{l}\text { Number of } \\
\text { times positive } \\
\text { blood cultures }\end{array}$} & \multirow[t]{4}{*}{$22.73 \pm 9.13$} & \multirow[t]{4}{*}{$39.73 \pm 18.27$} & \multirow[t]{4}{*}{ NA } & \multirow[t]{4}{*}{ NA } \\
\hline & ITT: Yes & & & & & & & & & \\
\hline & $\begin{array}{l}\text { Blinding: } \\
\text { Single-blind } \\
\text { (outcomes) }\end{array}$ & & & & & & & & & \\
\hline & 8 & & & & $0.20 \pm 0.41$ & $0.73 \pm 0.96$ & & & & \\
\hline
\end{tabular}


Table 4 Relevant outcome parameters of included randomized studies of enteral glutamine supplementation in critically ill patients (Continued)

\begin{tabular}{|c|c|c|c|c|c|c|c|c|c|c|}
\hline \multirow{8}{*}{$\begin{array}{l}\text { van Zanten } \\
\text { et al. [14] }\end{array}$} & C. random: Yes & \multirow{8}{*}{$\begin{array}{l}0.28 \text { (mean intake) glutamine, } \\
\text { omega-3, antioxidant-enriched } \\
\text { EN (experimental product) vs. } \\
\text { isonitrogenous, isocaloric } \\
\text { high-protein EN (Nutrison } \\
\text { Advanced Protison; Nutricia } \\
\text { Advanced Medical Nutrition, } \\
\text { Amsterdam, the Netherlands) }\end{array}$} & Hospital & Hospital & \multirow[t]{8}{*}{$80 / 152(53)$} & \multirow[t]{8}{*}{$78 / 149(52)$} & \multirow[t]{8}{*}{$38.2 \pm 28.9$} & \multirow[t]{8}{*}{$37.7 \pm 27.5$} & \multirow[t]{8}{*}{$23.7 \pm 22.4$} & \multirow[t]{8}{*}{$25.6 \pm 24.0$} \\
\hline & ITT: Yes & & $38 / 152(25)$ & $33 / 149(22)$ & & & & & & \\
\hline & $\begin{array}{l}\text { Blinding: } \\
\text { Double-blind }\end{array}$ & & $\mathrm{ICU}$ & $\mathrm{ICU}$ & & & & & & \\
\hline & \multirow[t]{5}{*}{12} & & $30 / 152(20)$ & $29 / 149(20)$ & & & & & & \\
\hline & & & 28 days & 28 days & & & & & & \\
\hline & & & $31 / 152(20)$ & 25/149 (17) & & & & & & \\
\hline & & & 6 months & 6 months & & & & & & \\
\hline & & & $53 / 152(35)$ & 42/149 (29) & & & & & & \\
\hline \multirow{8}{*}{$\begin{array}{l}\text { van Zanten } \\
\text { et al. [14] } \\
\text { trauma } \\
\text { subgroup }\end{array}$} & C. random: Yes & \multirow{8}{*}{$\begin{array}{l}0.28 \text { (mean intake) glutamine, } \\
\text { omega-3, antioxidant-enriched } \\
\text { EN (experimental product) vs. } \\
\text { isonitrogenous, isocaloric } \\
\text { high-protein EN (Nutrison } \\
\text { Advanced Protison) }\end{array}$} & Hospital & Hospital & \multirow[t]{8}{*}{ 32/55 (58) } & \multirow[t]{8}{*}{$36 / 54$ (67) } & \multirow[t]{8}{*}{$44.4 \pm 31.2$} & \multirow[t]{8}{*}{$39.8 \pm 25.3$} & \multirow[t]{8}{*}{$31.3 \pm 30.3$} & \multirow[t]{8}{*}{$32.5 \pm 27.5$} \\
\hline & ITT: Yes & & 6/55 (11) & 6/54 (11) & & & & & & \\
\hline & $\begin{array}{l}\text { Blinding: } \\
\text { Double-blind }\end{array}$ & & ICU & ICU & & & & & & \\
\hline & \multirow[t]{5}{*}{12} & & $5 / 55(9)$ & $6 / 54(11)$ & & & & & & \\
\hline & & & 28 days & 28 days & & & & & & \\
\hline & & & $4 / 55(7)$ & $2 / 54(4)$ & & & & & & \\
\hline & & & 6 months & 6 months & & & & & & \\
\hline & & & 8/55 (15) & $59 / 54(17)$ & & & & & & \\
\hline
\end{tabular}

C. random concealed randomization median (range), EN enteral nutrition, ITT intent to treat, $I V$ intravenous, $N A$ not applicable, $N J$ nasojejunal, TPN total parenteral nutrition Data are presented as mean \pm standard deviation or number (\%), as appropriate

a Hospital mortality unless otherwise stated

${ }^{\mathrm{b}}$ Number of patients with infections unless otherwise stated

${ }^{c}$ Median and range hence not included in meta-analysis (Hall et al. 2003 [27]; $p=$ not significant)

dSubgroup of patients, hence not included in the meta-analyses [28]

eData from parenteral glutamine group not shown here 


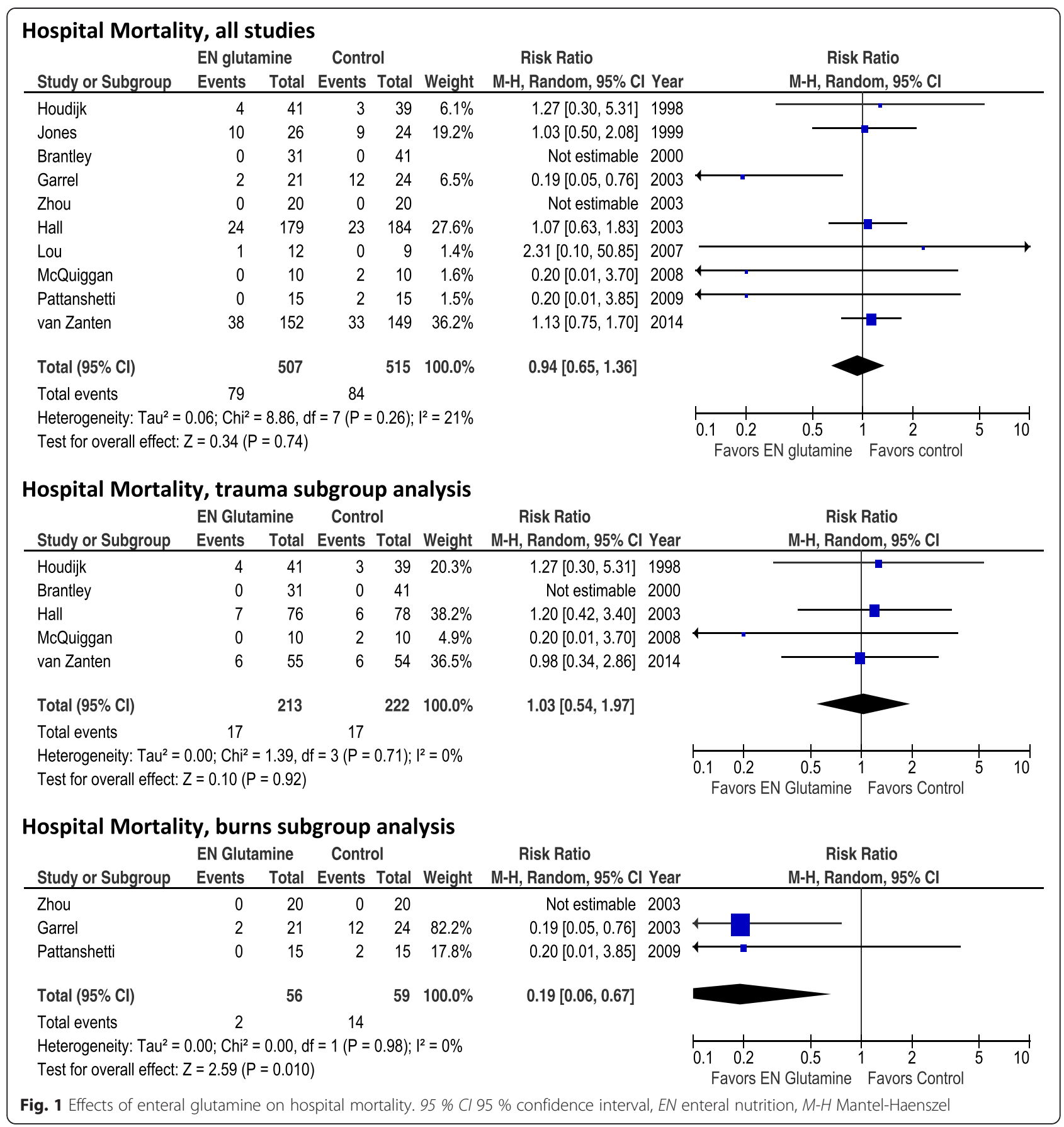

was not found to be significant for any of the endpoints, including hospital mortality $(p=0.18)$, infectious complications $(p=0.23)$, ICU LOS $(p=0.25)$ and hospital LOS $(p=0.48)$.

\section{Discussion}

Our overall results reveal that enteral GLN does not confer reductions in hospital mortality, with the exception of burn patients. We observed marked heterogeneity among the included studies, which are described in detail in Table 4. In our present analysis, we could not find strong signals of publication bias effects on the primary outcome parameter, hospital mortality. Whether this means that enteral GLN supplementation is safe for critically ill patients should be interpreted with caution, as previous analyses of parenteral GLN supplementation have demonstrated divergent effects on mortality when single-center studies were compared with multicenter trials; in other words, the observed beneficial effects on mortality were due to effects in single-center studies 


\begin{tabular}{|c|c|c|c|c|c|c|c|c|}
\hline \multicolumn{9}{|c|}{ Infectious complications, all studies } \\
\hline \multirow[b]{2}{*}{ Study or Subgroup } & \multicolumn{2}{|c|}{ EN glutamine } & \multicolumn{2}{|c|}{ Control } & \multicolumn{2}{|r|}{ Risk Ratio } & \multirow{2}{*}{\multicolumn{2}{|c|}{$\begin{array}{c}\text { Risk Ratio } \\
\text { M-H, Random, } 95 \% \mathrm{Cl}\end{array}$}} \\
\hline & Events & Total & Events & Total & Weight & M-H, Random, $95 \% \mathrm{Cl}$ Year & & \\
\hline Houdijk & 20 & 35 & 26 & 37 & $21.5 \%$ & $0.81[0.57,1.16] 1998$ & - & \\
\hline Zhou & 2 & 20 & 6 & 20 & $1.2 \%$ & $0.33[0.08,1.46] 2003$ & $\hookleftarrow$ & \\
\hline Hall & 38 & 179 & 43 & 184 & $18.4 \%$ & $0.91[0.62,1.33] 2003$ & & \\
\hline van Zanten & 80 & 152 & 78 & 149 & $58.8 \%$ & $1.01[0.81,1.25] 2014$ & & \\
\hline Total $(95 \% \mathrm{Cl})$ & & 386 & & 390 & $100.0 \%$ & $0.93[0.79,1.10]$ & & \\
\hline Total events & 140 & & 153 & & & & & \\
\hline $\begin{array}{l}\text { Heterogeneity: } \text { Tau }^{2}= \\
\text { Test for overall effect }\end{array}$ & $\begin{array}{l}0.00 ; \mathrm{Chi}^{2} \\
\mathrm{Z}=0.86(\mathrm{P}\end{array}$ & $\begin{array}{l}=2.94, \\
=0.39)\end{array}$ & $d f=3(P=$ & $=0.40)$; & $;\left.\right|^{2}=0 \%$ & & 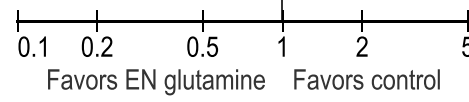 & $5 \quad 10$ \\
\hline \multicolumn{9}{|c|}{ Infectious complications, trauma subgroup analysis } \\
\hline & \multicolumn{2}{|c|}{ EN glutamine } & \multicolumn{2}{|c|}{ Control } & \multicolumn{2}{|r|}{ Risk Ratio } & \multirow{2}{*}{\multicolumn{2}{|c|}{$\begin{array}{c}\text { Risk Ratio } \\
\text { M-H, Random, } 95 \% \mathrm{Cl}\end{array}$}} \\
\hline Study or Subgroup & Events & Total & Events & Total & Weight & M-H, Random, 95\% Cl Year & & \\
\hline Houdijk & 20 & 35 & 26 & 37 & $40.5 \%$ & $0.81[0.57,1.16] 1998$ & $-\mathbf{n}$ & \\
\hline van Zanten & 32 & 55 & 36 & 54 & $59.5 \%$ & $0.87[0.65,1.17] 2014$ & 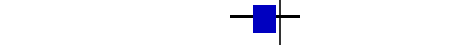 & \\
\hline Total $(95 \% \mathrm{Cl})$ & & 90 & & 91 & $100.0 \%$ & $0.85[0.68,1.06]$ & & \\
\hline Total events & 52 & & 62 & & & & & \\
\hline $\begin{array}{l}\text { Heterogeneity: } \mathrm{Tau}^{2} \\
\text { Test for overall effect }\end{array}$ & $\begin{array}{l}0.00 ; \mathrm{Chi}^{2}= \\
z=1.43(\mathrm{P}\end{array}$ & $\begin{array}{l}=0.09, c \\
=0.15)\end{array}$ & $d f=1(P=$ & $=0.76) ;$ & $1^{2}=0 \%$ & & 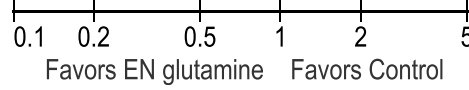 & $5 \quad 10$ \\
\hline
\end{tabular}

$[16,66]$. With respect to enteral GLN, our analysis included only one multicenter study, and the results of that study suggest increased harm [14].

\section{Lack of effect on infectious morbidity}

In contrast to earlier observations, we could not demonstrate any beneficial effect of enteral GLN on infectious morbidity. Only in burn patients was a small effect seen; however, the number of patients is limited, which precludes making strong recommendations. Recently, a lack of effect of GLN administration to boost the innate immune system response in trauma patients in the ICU has been demonstrated. No increase in the expression and/or functionality of Toll-like receptors, key receptors

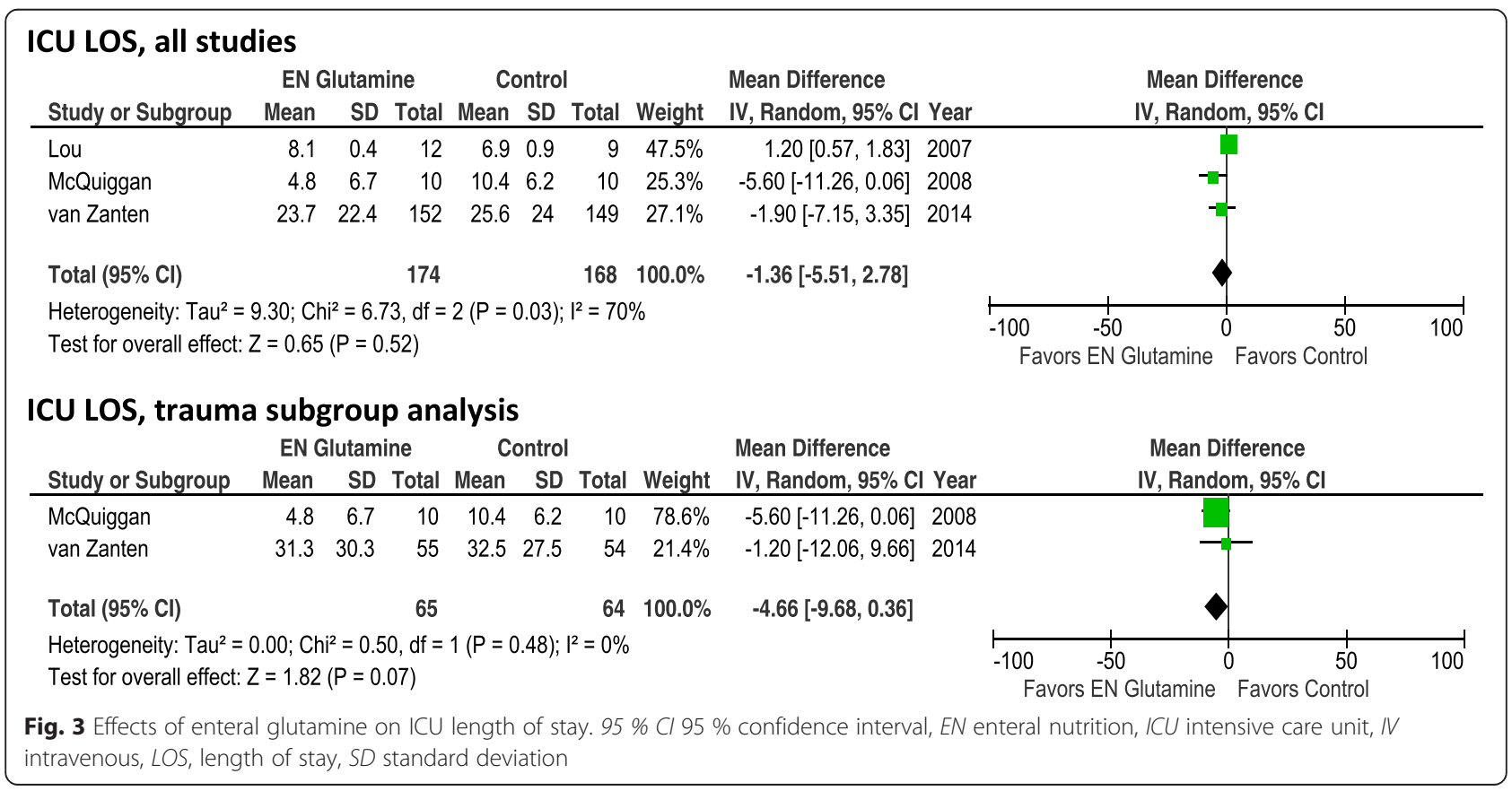




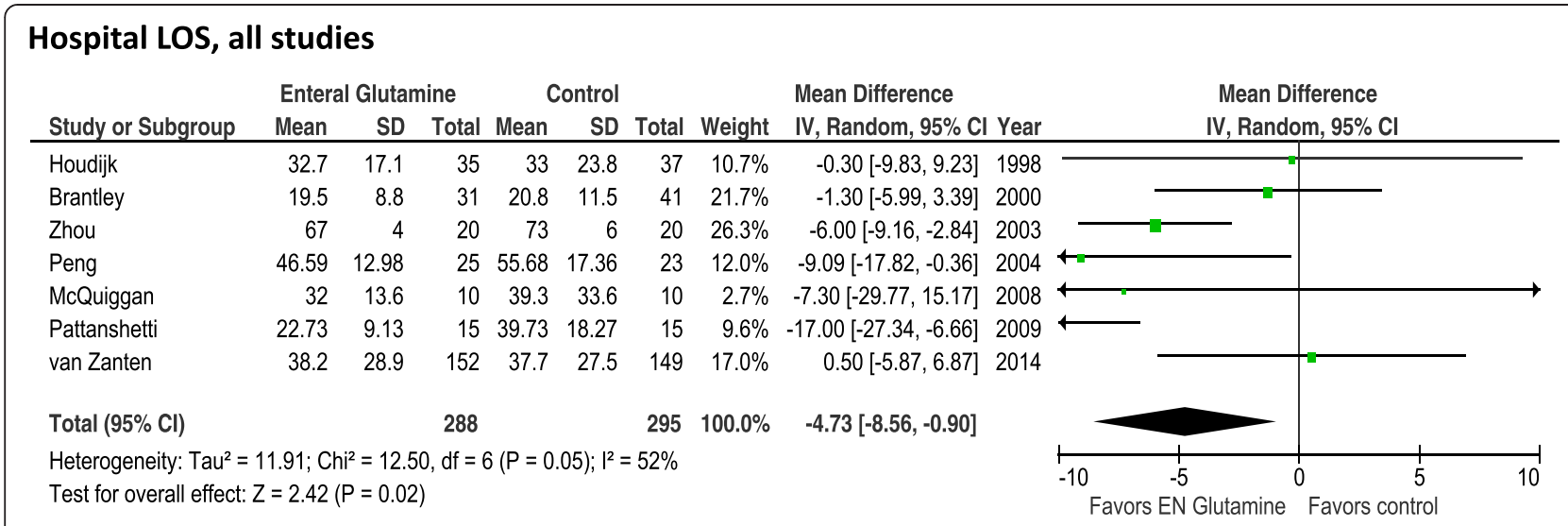

\section{Hospital LOS, trauma subgroup analysis}

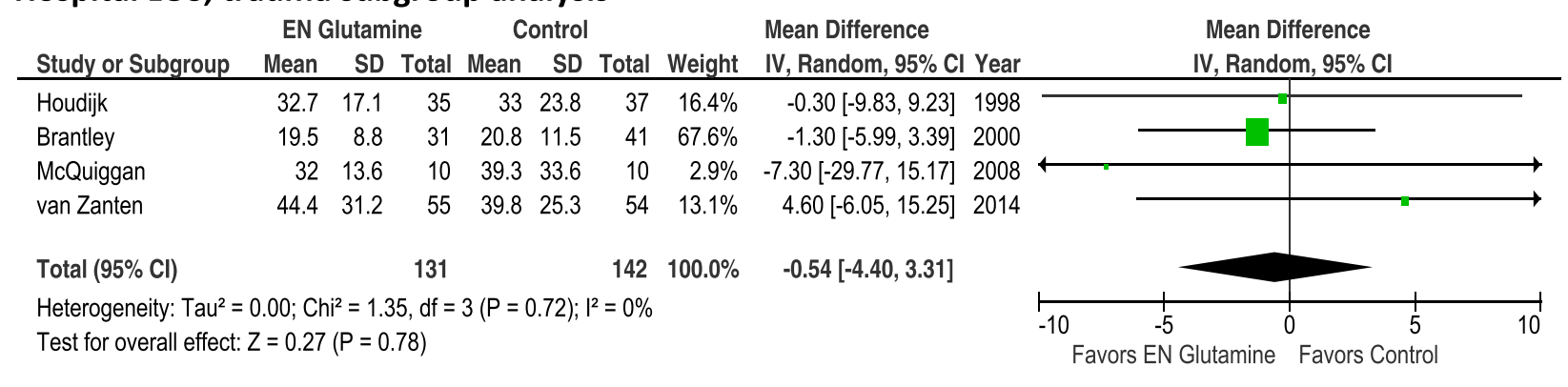

Hospital LOS, burns subgroup analysis

\begin{tabular}{|c|c|c|c|c|c|c|c|c|c|c|c|c|}
\hline \multirow[b]{2}{*}{ Study or Subgroup } & \multicolumn{3}{|c|}{ EN Glutamine } & \multicolumn{3}{|c|}{ Control } & \multicolumn{3}{|c|}{ Mean Difference } & \multirow{2}{*}{\multicolumn{3}{|c|}{$\begin{array}{c}\text { Mean Difference } \\
\text { IV, Random, } 95 \% \mathrm{Cl}\end{array}$}} \\
\hline & Mean & SD & Total & Mean & SD & Total & Weight & IV, Random, 95\% Cl & Year & & & \\
\hline Zhou & 67 & 4 & 20 & 73 & 6 & 20 & $52.4 \%$ & $-6.00[-9.16,-2.84]$ & 2003 & - & & \\
\hline Peng & 46.59 & 12.98 & 25 & 55.68 & 17.36 & 23 & $26.3 \%$ & $-9.09[-17.82,-0.36]$ & 2004 & $\leftarrow$ & & \\
\hline Pattanshetti & 22.73 & 9.13 & 15 & 39.73 & 18.27 & 15 & $21.3 \%$ & $-17.00[-27.34,-6.66]$ & 2009 & & & \\
\hline Total $(95 \% \mathrm{Cl})$ & & & 60 & & & 58 & $100.0 \%$ & $-9.16[-15.06,-3.26]$ & & & & \\
\hline \multicolumn{10}{|c|}{ Heterogeneity: $\mathrm{Tau}^{2}=14.70 ; \mathrm{Chi}^{2}=4.19, \mathrm{df}=2(P=0.12) ; \mathrm{I}^{2}=52 \%$} & -10 & 5 & 10 \\
\hline \multicolumn{10}{|c|}{ Test for overall effect: $Z=3.04(P=0.002)$} & Favors EN Glutamine & Favors Control & \\
\hline
\end{tabular}

Fig. 4 Effects of enteral glutamine on hospital length of stay. $95 \%$ Cl $95 \%$ confidence interval, EN enteral nutrition, IV intravenous, LOS, length of stay, SD standard deviation

that sense infections, was found in response to GLN supplementation [67]. Furthermore, protein intake and infectious morbidity seem to be associated [68]. Therefore, the effects of GLN supplementation can be studied adequately only in isonitrogenous intervention studies.

\section{Reduction of hospital length of stay}

Although a reduction in ICU LOS could not be demonstrated, a reduction in hospital LOS of approximately 4.7 days (WMD $-4.73,95 \%$ CI -8.56 to -0.90 ) persisted. These results are in line with previously reported metaanalyses $[17,53]$. This signal is driven largely by three studies of burn patients that, when aggregated, show a reduction of more than 9 days in the hospital.

\section{Glutamine in burn patients}

Burn patients may be a unique group of patients for which GLN has clinically significant beneficial effects. Low blood GLN levels have been observed in this patient population $[28,29]$, and the conditionally essential hypothesis may apply to them. As noted in this review, several small trials have shown benefits with regard to blood infections [28] and LOS [29]. A similar effect was observed on Gram-negative blood infections in two studies with different routes of GLN administration $[28,69]$. In a more recent study [33], researchers reported a decrease in blood infection of identical magnitude. Taken together, these observations strongly suggest that GLN has a significant effect on blood infections in burn patients. Decreased mortality was also found in one study with enteral GLN administration [28], 
although that trial had a high mortality rate in the control group. Although this study was not powered for testing an effect on mortality, the effect size observed warrants a larger trial. Such a large-scale, multicenter trial is currently underway, and stronger inferences about the use of GLN in burn patients awaits the results [70].

\section{Previous meta-analyses}

In two meta-analyses of the initial randomized controlled trials of enteral GLN supplementation, García-de-Lorenzo et al. [53] and Jiang et al. [17] reported data on 17 and 7 studies, respectively. Although in the first meta-analysis the number of studies that included various patient categories was larger than in our present analysis, the number of studies addressing the effects of enteral GLN in critically ill patients was much lower. On the basis of their results, although the doses given and the duration of therapy varied widely depending on the pathologic condition, García-de-Lorenzo et al. [53] recommended using GLN intake of 20-30 g/day, early initiation of diet and maintenance for at least 5 days (grade $\mathrm{C}$ recommendations) [53]. Jiang et al. [17] concluded that administration of GLNenhanced EN in patients with critical illness may reduce nosocomial infection rates and shorten hospital LOS. Furthermore, they recommended that studies be done with a large sample size to verify the efficiency of GLN-enhanced EN on lowering mortality in patients with critical illness. Since the publication of the García-de-Lorenzo et al. and Jiang et al. studies, another four studies have been published, including the multinational, multicenter MetaPlus study [14]. Incorporating data of these studies enabled us to evaluate GLN in more than 1000 patients.

\section{Safety concerns regarding glutamine supplementation} Two large multicenter trials, the REDOXS and the MetaPlus trials [13, 14], have fueled the debate on the safety and efficacy of GLN supplementation. Therefore, we believe they need to be discussed in more detail. The REDOXS trial was not included in our present analysis for reasons of both enteral and parenteral GLN supplementation [13].

The REDOXS trial was a factorial $2 \times 2$ randomized trial conducted in 40 ICUs in North America and Europe. A total of 1223 mechanically ventilated adult patients with multiple organ dysfunction syndrome were randomized to receive high doses of GLN, antioxidants, both or placebo separate from artificial nutrition [13]. Total caloric intake in both groups was about $900 \mathrm{kcal} /$ day. The primary analysis demonstrated no clinical benefit and identified a trend toward increased mortality at 28 days (32.4\% vs. $27.2 \%$; adjusted odds ratio 1.28; $95 \%$ CI 1.00-1.64; $p=0.049$ ) and a significant increase in hospital and 6-month mortality among patients who received GLN.
There was no effect of antioxidants on 28-day mortality [13].

The MetaPlus trial was conducted from February 2010 through April 2012. It included a 6-month follow-up period in 14 ICUs in the Netherlands, Germany, France and Belgium. A total of 301 adult patients who were expected to be ventilated and to require $\mathrm{EN}$ for more than $72 \mathrm{~h}$ were randomized to the intervention feed (enriched with GLN and with antioxidants including selenium and fish oils) or control feed within $48 \mathrm{~h}$ of ICU admission and continued during ICU stay [14]. Consistent with attempting to supplement patients because of presumed nutrient deficiency, per $1500 \mathrm{ml}$, the enriched diet contained $113 \mathrm{~g}$ of protein, of which $23 \mathrm{~g}$ were alanylglutamine, and a total GLN content of $30 \mathrm{~g}$; relatively high amounts of antioxidants, including $285 \mu \mathrm{g}$ of selenium and an additional $7.5 \mathrm{~g}$ of fish oils. The control group received an isocaloric standard high-protein EN diet with similar amounts of proteins. There were no statistically significant differences in new infections according to the Centers for Disease Control and Prevention (CDC) definitions: $53 \%$ (95 \% CI 44-61 \%) in the enriched group versus $52 \%$ (95\% CI 44-61\%) in the control group $(p=0.96)$. The study was designed to observe a $50 \%$ relative reduction in incidence of new infections based on an incidence of $25 \%$ in the control arm (absolute reduction of $12.5 \%)$. The actual incidence of infections was larger than estimated (53\% and $52 \%$ ). Therefore, the study was not underpowered to detect differences in infections. Although the incidence of infections was higher than estimated, no reduction of infectious morbidity was observed. Secondary endpoints included mortality, Sequential Organ Failure Assessment scores, mechanical ventilation duration, ICU and hospital LOS and subtypes of infections according to CDC definitions. No differences were observed in secondary endpoints, except for a higher 6-month adjusted mortality rate in the enriched group (hazard ratio 1.57, 95 \% CI 1.03$2.39 ; p=0.04$ ) and an unadjusted higher mortality of $54 \%$ in the medical subgroup (95 \% CI 40-67\%) versus $35 \%$ (95 \% CI 22-49\%) ( $p=0.04)$. Mortality was a secondary endpoint in this study. However, we cannot ignore the observation of increased mortality just because it was a secondary endpoint. We have to look at these secondary endpoints when considering the safety of the intervention [71].

This meta-analysis does not suggest increased mortality with the use of enteral GLN supplementation. The signals of harm in the REDOXS trial may be due to the high dose of both enteral and parenteral GLN used, the negative effects in patients with renal failure and the low total caloric and protein intake, although these factors remain speculative. In the MetaPlus study, harmful effects were observed in all patients with respect to the 
adjusted 6-month mortality and unadjusted in the medical subgroup. The underlying mechanisms are still unclear, but data suggest that patients did worse if their baseline GLN plasma levels were higher. Another explanation could be that effects are due to the other immune-modulating ingredients or to an interaction among those.

\section{Is the glutamine conditional deficiency hypothesis still valid?}

Overall, the benefits of enteral GLN supplementation seem to be limited. This should lead to a reevaluation of the importance and validity of the conditional deficiency hypothesis of GLN in critically ill patients. Some have suggested that low GLN plasma levels at ICU admission may be an adaptive response and that supplementation could be considered as a maladaptive response to this [72].

Several observations challenging the hypothesis have been published recently. The frequency of patients with low baseline plasma GLN levels is extremely variable and is not consistent $[31,73,74]$. There is no association of baseline plasma GLN and Acute Physiology and Chronic Health Evaluation II score, as could be expected when the severity of illness plays a role in conditional deficiency [74]. Moreover, in general and septic ICU patients, high baseline plasma GLN $(>930 \mu \mathrm{mol} / \mathrm{L})$ was associated with increased mortality suggesting a U-shaped association [74]. In addition, low baseline GLN levels were not always associated with increased mortality [73].

Considering conditional deficiency, the GLN rate of appearance from muscles to plasma is expected to be around the maximum production rate, estimated by isotopic techniques at $40-80 \mathrm{~g} / 24 \mathrm{~h}$ [75]. However, this maximum muscle output could not be confirmed in a tracer study in ICU patients. The endogenous production of GLN in muscles and appearance in plasma were related to severity of illness and did not diminish by supplementation of GLN [76]. A trend toward higher mortality was demonstrated in patients with higher baseline GLN levels treated with GLN-enriched EN [14]. After ICU discharge, patients showed normalized plasma GLN levels not associated with long-term outcome. However, patients with the highest plasma GLN on the ICU discharge day showed a higher 1-year mortality [77].

\section{Consequences of findings}

Our observations do not support use of GLN in critically ill patients; therefore, our present systematic review and meta-analysis is important. Moreover, the recent largescale, multicenter trials (REDOXS and MetaPlus) show no benefits, but instead indicate signals of increased harm with respect to long-term mortality. All these observations and the concerns that have been published should lead to a reevaluation of the validity of the GLN hypothesis in critically ill patients and probably also to new recommendations for the practice guidelines developed by organizations such as the European Society for Clinical Nutrition and Metabolism, the American Society of Parenteral and Enteral Nutrition and the Canadian Practice Guidelines Committee [15].

\section{Strengths and weaknesses}

The strengths of our meta-analysis include the use of several methods to reduce bias: a comprehensive search of the worldwide literature, including trials published in languages other than English; duplicate data abstraction; specific criteria for searching and analysis; and no industry funding. We also focused on clinically important primary outcomes. Furthermore, we created funnel plots for all primary and key secondary endpoints examined to look for possible publication bias associated with these endpoints.

In contrast, we are aware that our meta-analysis has several limitations. Among these are the limited number of larger trials $[14,27]$ and the small number of trials included in certain subgroup analyses. Owing to the heterogeneity of the included studies, the internal validity of our findings should be interpreted with caution. We also unfortunately could not perform subgroup analysis for all endpoints, owing to the limited number of trials in which the particular endpoints were examined. Another potential weakness of our review is that the studies included were published over the course of 2 decades. This may be relevant, as in a time-sequential analysis, Fadda and coworkers studied the effects of GLN supplementation over time. They showed that only trials performed before 2003 manifested a positive signal, whereas more recent trials failed to demonstrate any positive treatment effect [78]. Hence, it appears that only older, small, single-center trials of intravenous GLN, when meta-analyzed, showed a positive treatment effect.

\section{Conclusions}

In this comprehensive systematic review, we demonstrate that enteral GLN supplementation given in conjunction with EN support does not confer significant reductions in hospital mortality among critically ill patients, including trauma patients. However, it may reduce hospital mortality in burn patients. No effects on infectious morbidity or ICU LOS were observed. Hospital LOS was significantly reduced in critically ill and burn patients but not in trauma patients. However, the results of our meta-analysis are based mainly on smaller, single-center studies, and two recent multicenter trials have suggested potential harm of GLN. Therefore, enteral GLN supplementation cannot be recommended for critically ill patients. In burn patients, larger studies are warranted, as our observations of a beneficial effect are 
based on a small number of patients. Such a trial is currently underway worldwide (citation: see Clinical trials. gov ID \#NCT00985205).

\section{Key messages}

- In critically ill patients, including trauma patients, supplemental enteral GLN does not decrease hospital mortality, infectious morbidity or ICU LOS.

- Supplemental enteral GLN does significantly reduce hospital mortality in burn patients; however, the relevant studies were small.

- Supplemental enteral GLN significantly shortens hospital LOS in critically ill and burn patients but not in critically ill trauma patients.

- Supplemental enteral GLN should not be given to critically ill patients or trauma patients, as its benefits are limited.

- Moreover, results are based mainly on single-center studies, and two recent multicenter trials have suggested potential harm of GLN.

- More data on enteral GLN supplementation are warranted in burn patients as present observations of a benefit are based on a small number of patients.

\section{Abbreviations}

CDC: Centers for Disease Control and Prevention; EN: Enteral nutrition; GLN: Glutamine; ICU: Intensive care unit; ITT: Intention to treat: IV: Intravenous; LOS: Length of stay; M-H: Mantel-Haenszel; NA: Not applicable; NJ: Nasojejunal; PN: Parenteral nutrition; RCT: Randomized controlled trial; REDOXS: Reducing Deaths Due to Oxidative Stress Trial; RevMan: Review Manager software; RR: Risk ratio; SD: Standard deviation; TPN: Total parenteral nutrition; WMD: Weighted mean difference.

\section{Competing interests}

No funding for the development, writing or submission of this manuscript for publication was received. ARHVZ received research funding for the MetaPlus trial of glutamine and antioxidants from Nutricia. DKH received research funding for the REDOXS trial of glutamine and antioxidants from Fresenius Kabi. RD and DG declare that they have no competing interests.

\section{Authors' contributions}

ARHvZ contributed to the development of the review concept, study grading, study selection and evaluation and interpretation of data, and also was the primary author and editor of the manuscript. RD contributed to the development of the review concept, study grading, study selection and evaluation and interpretation of data; performed much of the primary statistical analysis and meta-analysis of data; and contributed significantly to the writing and editing of all drafts of the manuscript. DG contributed to the development of the review concept, study grading, study evaluation and interpretation of the data. DKH contributed to the development of the review concept, study grading, study selection and evaluation and interpretation of data, and also assisted in primary editing of all drafts of the manuscript. All authors read and approved the final manuscript.

\section{Acknowledgments}

The authors acknowledge Margot Lemieux and Xuran Jiang (biostatistician) for assistance with statistical analysis and figure generation for this article.

\section{Author details}

'Department of Intensive Care Medicine, Gelderse Vallei Hospital, Willy Brandtlaan 10, 6716 RP Ede, The Netherlands. ${ }^{2}$ Clinical Evaluation Research Unit, Kingston General Hospital, Angada 4, 76 Stuart Street, Kingston, ON K7L 2V7, Canada. ${ }^{3}$ Department of Nutrition, University of Montreal, Pavillon
Liliane de Stewart, 2405, Chemin de la Côte-Sainte-Catherine, Local 1204, Montreal, QC H3T 1A8, Canada.

Received: 2 March 2015 Accepted: 17 July 2015

Published online: 18 August 2015

\section{References}

1. Hegazi RA, Wischmeyer PE. Clinical review: optimizing enteral nutrition for critically ill patients - a simple data-driven formula. Crit Care. 2011;15:234.

2. Wernerman J. Glutamine supplementation to critically ill patients? Crit Care. 2014;18:214

3. Cruzat VF, Bittencourt A, Scomazzon SP, Leite JS, de Bittencourt PI, Tirapegui $\mathrm{Jr} \mathrm{J}$. Oral free and dipeptide forms of glutamine supplementation attenuate oxidative stress and inflammation induced by endotoxemia. Nutrition. 2014;30:602-11.

4. Oudemans-van Straaten HM, Bosman RJ, Treskes M, van der Spoel HJ, Zandstra DF. Plasma glutamine depletion and patient outcome in acute ICU admissions. Intensive Care Med. 2001;27:84-90.

5. Griffiths RD, Jones C, Palmer TE. Six-month outcome of critically ill patients given glutamine-supplemented parenteral nutrition. Nutrition. 1997;13:295-302.

6. Vanek W, Matarese LE, Robinson M, Sacks GS, Young LS, Kochevar M, et al. A.S.P.E.N. position paper: parenteral nutrition glutamine supplementation. Nutr Clin Pract. 2011;26:479-94.

7. Montejo JC, Zarazaga A, Lopez-Martinez J, Urrútia G, Roqué M, Blesa AL, et al. Immunonutrition in the intensive care unit: a systematic review and consensus statement. Clin Nutr. 2003;22:221-33.

8. Avenell A. Glutamine in critical care: current evidence from systematic reviews. Proc Nutr Soc. 2006;65:236-41.

9. McClave SA, Martindale RG, Vanek WW, McCarthy M, Roberts P, Taylor B, et al. Guidelines for the provision and assessment of nutrition support therapy in the adult critically ill patient: Society of Critical Care Medicine (SCCM) and American Society for Parenteral and Enteral Nutrition (A.S.P.E.N.). JPEN Parenter Enteral Nutr. 2009;33:277-316.

10. Heyland DK, Dhaliwal R, Drover JW, Gramlich L, Dodek P, Canadian Critical Care Clinical Practice Guidelines Committee. Canadian clinical practice guidelines for nutrition support in mechanically ventilated, critically ill adult patients. JPEN Parenter Enteral Nutr. 2003;27:355-73.

11. Kreymann KG, Berger MM, Deutz NE, Hiesmayr M, Jolliet P, Kazandjiev G, et al. ESPEN guidelines on enteral nutrition: intensive care. Clin Nutr. 2006;25:210-23.

12. Tao KM, Li XQ, Yang LQ, Yu WF, Lu ZJ, Sun YM, et al. Glutamine supplementation for critically ill adults. Cochrane Database Syst Rev. 2014;9:CD010050.

13. Heyland D, Muscedere J, Wischmeyer PE, Cook D, Jones G, Albert M, et al. A randomized trial of glutamine and antioxidants in critically ill patients. N Engl J Med. 2013;368:1489-97. A published erratum appears in N Engl J Med. 2013;368:1853 [dosage error in original text].

14. van Zanten AR, Sztark F, Kaisers UX, Zielmann S, Felbinger TW, Sablotzki AR, et al. High-protein enteral nutrition enriched with immune-modulating nutrients vs standard high-protein enteral nutrition and nosocomial infections in the ICU: a randomized clinical trial. JAMA. 2014;312:514-24.

15. van Zanten AR, Hofman Z, Heyland DK. Consequences of the REDOXS and METAPLUS Trials: The End of an Era of Glutamine and Antioxidant Supplementation for Critically III Patients? JPEN J Parenter Enteral Nutr. 2015. pii: 0148607114567201 . [Epub ahead of print] PubMed PMID: 25567781

16. Wischmeyer PE, Dhaliwal R, McCall M, Ziegler TR, Heyland DK. Parenteral glutamine supplementation in critical illness: a systematic review. Crit Care. 2014;18:R76.

17. Jiang H, Chen W, Hu W, Cai B, Liao RJ. The impact of glutamine-enhanced enteral nutrition on clinical outcome of patients with critical illness: a systematic review of randomized controlled trials. Zhonghua Shao Shang Za Zhi. 2009;25:325-30. Chinese.

18. Melis GC, Boelens PG, van der Sijp JR, Popovici T, De Bandt JP, Cynober L, et al. The feeding route (enteral or parenteral) affects the plasma response of the dipeptide Ala-GIn and the amino acids glutamine, citrulline and arginine, with the administration of Ala-GIn in preoperative patients Br J Nutr. 2005;94:19-26.

19. Heyland DK, MacDonald S, Keefe L, Drover JW. Total parenteral nutrition in the critically ill patient: a meta-analysis. JAMA. 1998;280:2013-9. 
20. DerSimonian R, Laird N. Meta-analysis in clinical trials. Control Clin Trials. 1986;7:177-88.

21. The Cochrane Collaboration. Review Manager (RevMan) [computer program]. Version 5.3. The Nordic Cochrane Centre, The Cochrane Collaboration; 2014

22. Higgins JP, Thompson SG. Quantifying heterogeneity in a meta-analysis. Stat Med. 2002;21:1539-58.

23. Rucker $G$, Schwarzer $G$, Carpenter J. Arcsine test for publication bias in meta-analyses with binary outcomes. Stat Med. 2008;27:746-63.

24. Houdijk AP, Rijnsburger ER, Jansen J, Wesdorp RI, Weiss JK, McCamish MA, et al. Randomised trial of glutamine-enriched enteral nutrition on infectious morbidity in patients with multiple trauma. Lancet. 1998;352:772-6.

25. Jones C, Palmer TE, Griffiths RD. Randomized clinical outcome study of critically ill patients given glutamine-supplemented enteral nutrition. Nutrition. 1999;15:108-15.

26. Brantley S, Pierce J. Effects of enteral glutamine on trauma patients [abstract]. Nutr Clin Pract. 2000;15:S13.

27. Hall JC, Dobb G, Hall J, De Sousa R, Brennan L, McCauley R. A prospective randomized trial of enteral glutamine in critical illness. Intensive Care Med. 2003:29:1710-6.

28. Garrel D, Patenaude J, Nedelec B, Samson L, Dorais J, Champoux J, et al. Decreased mortality and infectious morbidity in adult burn patients given enteral glutamine supplements: a prospective, controlled, randomized clinical trial. Crit Care Med. 2003;31:2444-9.

29. Zhou YP, Jiang ZM, Sun YH, Wang XR, Ma EL, Wilmore D. The effect of supplemental enteral glutamine on plasma levels, gut function, and outcome in severe burns: a randomized, double-blind, controlled clinical trial. JPEN J Parenter Enteral Nutr. 2003;27:241-5.

30. Peng $X$, Yan $H$, You Z, Wang P, Wang S. Effects of enteral supplementation with glutamine granules on intestinal mucosal barrier function in severe burned patients. Burns. 2004;30:135-9.

31. Luo M, Bazargan N, Griffith DP, Estívariz CF, Leader LM, Easley KA, et al. Metabolic effects of enteral versus parenteral alanyl-glutamine dipeptide administration in critically ill patients receiving enteral feeding: a pilot study. Clin Nutr. 2008;27:297-306

32. McQuiggan M, Kozar R, Sailors RM, Ahn C, McKinley B, Moore F. Enteral glutamine during active shock resuscitation is safe and enhances tolerance of enteral feeding. JPEN J Parenter Enteral Nutr. 2008;32:28-35.

33. Pattanshetti VM, Powar RS, Godhi AS, Metgud SC. Enteral glutamine supplementation reducing infectious morbidity in burns patients: a randomised controlled trial. Indian J Surg. 2009;71:193-7.

34. Jebb SA, Marcus R, Elia M. A pilot study of oral glutamine supplementation in patients receiving bone marrow transplants. Clin Nutr. 1995;14:162-16.

35. Long CL, Nelson KM, DiRienzo DB, Weis JK, Stahl RD, Broussard TD, et al. Glutamine supplementation of enteral nutrition: impact on whole body protein kinetics and glucose metabolism in critically ill patients. JPEN J Parenter Enteral Nutr. 1995;19:470-6.

36. Jensen GL, Miller RH, Talabiska DG, Fish J, Gianferante L. A double-blind, prospective, randomized study of glutamine-enriched compared with standard peptide-based feeding in critically ill patients. Am J Clin Nutr. 1996;64:615-21.

37. Fish J, Sporay G, Beyer K, Jones J, Kihara T, Kennedy A, et al. A prospective randomized study of glutamine-enriched parenteral compared with enteral feeding in postoperative patients. Am J Clin Nutr. 1997;65:977-83.

38. Scolapio JS, Camilleri M, Fleming CR, Oenning LV, Burton DD, Sebo TJ, et al. Effect of growth hormone, glutamine, and diet on adaptation in shortbowel syndrome: a randomized, controlled study. Gastroenterology. 1997;113:1074-81.

39. Anderson PM, Ramsay NK, Shu XO, Rydholm N, Rogosheske J, Nicklow R, et al. Effect of low-dose oral glutamine on painful stomatitis during bone marrow transplantation. Bone Marrow Transplant. 1998;22:339-44.

40. Anderson PM, Schroeder G, Skubitz KM. Oral glutamine reduces the duration and severity of stomatitis after cytotoxic cancer chemotherapy. Cancer. 1998:83:1433-9.

41. Den Hond E, Hiele M, Peeters M, Ghoos Y, Rutgeerts P. Effect of long-term oral glutamine supplements on small intestinal permeability in patients with Crohn's disease. JPEN J Parenter Enteral Nutr. 1999;23:7-11.

42. Schloerb PR, Skikne BS. Oral and parenteral glutamine in bone marrow transplantation: a randomized, double-blind study. JPEN J Parenter Enteral Nutr. 1999;23:117-22.

43. Scolapio JS. Effect of growth hormone, glutamine, and diet on body composition in short bowel syndrome: a randomized, controlled study. JPEN J Parenter Enteral Nutr. 1999;23:309-12.
44. Zhou Y, Jiang Z, Sun Y. Glutamine dipeptide enriched enteral nutrition improving gut permeability in severe burns. Zhonghua Yi Xue Za Zhi. 1999;79:825-7. Chinese.

45. Jackson NC, Carroll PV, Russell-Jones DL, Sönksen PH, Treacher DF, Umpleby AM. Effects of glutamine supplementation, GH, and IGF-I on glutamine metabolism in critically ill patients. Am J Physiol Endocrinol Metab. 2000;278:E226-33.

46. Szkudlarek J, Jeppesen PB, Mortensen PB. Effect of high dose growth hormone with glutamine and no change in diet on intestinal absorption in short bowel patients: a randomised, double blind, crossover, placebo controlled study. Gut. 2000;47:199-205.

47. Chen $G$, Xie W, Jiang H. Clinical observation of the protective effect of oral feeding of glutamine granules on intestinal mucous membrane. Zhonghua Shao Shang Za Zhi. 2001;17:210-1. Chinese.

48. Scolapio JS, McGreevy K, Tennyson GS, Burnett OL. Effect of glutamine in short-bowel syndrome. Clin Nutr. 2001;20:319-23.

49. Velasco N, Hernandez G, Wainstein C, Castillo L, Maiz A, Lopez F, et al. Influence of polymeric enteral nutrition supplemented with different doses of glutamine on gut permeability in critically ill patients. Nutrition. 2001;17:907-11.

50. Boelens PG, Houdijk AP, Fonk JC, Nijveldt RJ, Ferwerda CC, Von Blomberg-Van Der Flier BM, et al. Glutamine-enriched enteral nutrition increases HLA-DR expression on monocytes of trauma patients. J Nutr. 2002;132:2580-6

51. Novak F, Heyland DK, Avenell A, Drover JW, Su X. Glutamine supplementation in serious illness: a systematic review of the evidence. Crit Care Med. 2002;30:2022-9.

52. Fläring UB, Rooyackers $\mathrm{OE}$, Wernerman J, Hammarqvist F. Glutamine attenuates post-traumatic glutathione depletion in human muscle. Clin Sci (Lond). 2003;104:275-82.

53. García-de-Lorenzo A, Zarazaga A, García-Luna PP, Gonzalez-Huix F, López-Martínez J, Miján A, et al. Clinical evidence for enteral nutritional support with glutamine: a systematic review. Nutrition. 2003;19:805-11.

54. Boelens PG, Houdijk AP, Fonk JC, Puyana JC, Haarman HJ, von Blomberg-van der Flier ME, et al. Glutamine-enriched enteral nutrition increases in vitro interferon-gamma production but does not influence the in vivo specific antibody response to KLH after severe trauma: a prospective, double blind, randomized clinical study. Clin Nutr. 2004;23:391-400.

55. Falcao de Arruda IS, de Aguilar-Nascimento JE. Benefits of early enteral nutrition with glutamine and probiotics in brain injury patients. Clin Sci (Lond). 2004;106:287-92.

56. Peng X, Yan H, You Z, Wang P, Wang S. Clinical and protein metabolic efficacy of glutamine granules-supplemented enteral nutrition in severely burned patients. Burns. 2005;31:342-6.

57. Peng X, Yan H, You Z, Wang P, Wang S. Glutamine granule-supplemented enteral nutrition maintains immunological function in severely burned patients. Burns. 2006:32:589-93.

58. Guo GH, Deng ZY, Wang YX, Xing JJ, Peng Y, Li GH. Effects of glutamine enriched enteral feeding on immunoregulation in burn patients. Zhonghua Shao Shang Za Zhi. 2007;23:406-8. Chinese.

59. Kuhls DA, Rathmacher JA, Musngi MD, Frisch DA, Nielson J, Barber A, et al. $\beta$-Hydroxy- $\beta$-methylbutyrate supplementation in critically ill trauma patients. J Trauma. 2007:62:125-31. discussion 131-2.

60. Spindler-Vesel A, Bengmark S, Vovk I, Cerovic O, Kompan L. Synbiotics, prebiotics, glutamine, or peptide in early enteral nutrition: a randomized study in trauma patients. JPEN J Parenter Enteral Nutr. 2007:31:119-26.

61. Beale RJ, Sherry T, Lei K, Campbell-Stephen L, McCook J, Smith J, et al. Early enteral supplementation with key pharmaconutrients improves Sequential Organ Failure Assessment score in critically ill patients with sepsis: outcome of a randomized, controlled, double-blind trial. Crit Care Med. 2008:36:131-44.

62. Han YY, Lai SL, Ko WJ, Chou CH, Lai HS. Effects of fish oil on inflammatory modulation in surgical intensive care unit patients. Nutr Clin Pract. 2012;27:91-8.

63. Cavalcante AA, Campelo MW, de Vasconcelos MP, Ferreira CM, Guimarães $\mathrm{SB}$, Garcia JH, et al. Enteral nutrition supplemented with L-glutamine in patients with systemic inflammatory response syndrome due to pulmonary infection. Nutrition. 2012;28:397-402.

64. Han W, Sun J, Han R, Wang Y, Qing Y, Li H, et al. Application of enteral nutrition support with different doses of glutamine in elderly critically ill patients. Chin J Clin Nutr. 2014;22:149-53. Chinese. 
65. Koksal GM, Erbabacan E, Tunali Y, Karaoren G, Vehid S, Oz H. The effects of intravenous, enteral and combined administration of glutamine on malnutrition in sepsis: a randomized clinical trial. Asia Pac J Clin Nutr. 2014:23:34-40.

66. Pasin L, Landoni G, Zangrillo A. Glutamine and antioxidants in critically ill patients. N Engl J Med. 2013;369:482-4.

67. Pérez-Bárcena J, Crespí C, Regueiro V, Marsé P, Raurich JM, Ibáñez J, et al. Lack of effect of glutamine administration to boost the innate immune system response in trauma patients in the intensive care unit. Crit Care. 2010;14:R233.

68. Weijs PJ. Fundamental determinants of protein requirements in the ICU. Curr Opin Clin Nutr Metab Care. 2014;17:183-9.

69. Wischmeyer PE, Lynch J, Liedel J, Wolfson R, Riehm J, Gottlieb L, et al. Glutamine administration reduces Gram-negative bacteremia in severely burned patients: a prospective, randomized, double-blind trial versus isonitrogenous control. Crit Care Med. 2001;29:2075-80.

70. Effects of Enteral Glutamine Supplementation on Mortality and Infectious Morbidity in Severely Burned Patients: a Multi-center Pilot Trial. ClinicalTrials.gov identifier: NCT00985205. https://clinicaltrials.gov/ct2/ results?term=nct00985205\&Search=Search. Accessed 4 August 2015.

71. van Zanten AR, Hofman Z. Standard vs enriched high protein enteral nutrition in the ICU—reply. JAMA. 2014;312:2288-9.

72. Van den Berghe G. Low glutamine levels during critical illness-adaptive or maladaptive? N Engl J Med. 2013;368:1549-50.

73. Hirose T, Shimizu K, Ogura H, Tasaki O, Hamasaki T, Yamano S, et al. Altered balance of the aminogram in patients with sepsis - the relation to mortality. Clin Nutr. 2014;33:179-82.

74. Rodas PC, Rooyackers O, Hebert C, Norberg $\AA$, Wernerman J. Glutamine and glutathione at ICU admission in relation to outcome. Clin Sci (Lond) 2012;122:591-7.

75. Wernerman J. Clinical use of glutamine supplementation. J Nutr 2008;138:2040S-4

76. Mori M, Rooyackers O, Smedberg M, Tjäder I, Norberg Å, Wernerman J. Endogenous glutamine production in critically ill patients: the effect of exogenous glutamine supplementation. Crit Care. 2014;18:R72.

77. Smedberg M, Grass JN, Pettersson L, Norberg $\AA$, Rooyackers O, Wernerman J. Plasma glutamine concentration after intensive care unit discharge: an observational study. Crit Care. 2014;18:677.

78. Fadda V, Maratea D, Trippoli S, Messori A. Temporal trend of short-term mortality in severely ill patients receiving parenteral glutamine supplementation. Clin Nutr. 2013;32:492-3.

\section{Submit your next manuscript to BioMed Central and take full advantage of:}

- Convenient online submission

- Thorough peer review

- No space constraints or color figure charges

- Immediate publication on acceptance

- Inclusion in PubMed, CAS, Scopus and Google Scholar

- Research which is freely available for redistribution 